\title{
Community Development Model for Public Health Applications: Overview of a Model to Eliminate Population Disparities
}

Robert G. Robinson, Dr. PH

For well over two decades, the public health community has undertaken a broad range of initiatives to identify and eliminate various health-related disparities among populations. The Centers for Disease Control and Prevention's (CDC) Office on Smoking and Health (OSH), for example, has committed resources to help states eliminate population disparities related to tobacco use. These initiatives have enjoyed a degree of success and some measurable decreases in population disparities. However, traditional public health approaches that are overly influenced by reductionist paradigms more content with risk factor assessment of at-risk strata may not be sufficient to produce successful results when applied to more intractable disparities. The elimination of disparities will require a more encompassing and comprehensive approach that addresses both population strata at risk and the communities in which they reside. This article proposes a new, concentrated model to address the elimination of population disparities-a model that focuses on community as the critical unit of analysis and action to achieve success.

Keywords: disparity; race; community; sexual orientation; social structure; racism; socioeconomic status; ethnicity; competence; development

The 21st Century should be the "century of the community" and the emphasis of efforts to improve theory and practice ought to reflect this paradigm. —Robinson (2004)

$\mathrm{T}$ The public health community has committed itself to defining and eliminating population disparities (U.S. Department of Health and Human Services, 2000). Many public health programs have long

Health Promotion Practice

July 2005 Vol. 6, No. 3, 338-346

DOI: $10.1177 / 1524839905276036$

(C2005 Society for Public Health Education included reducing disparities among their stated objectives and have launched specific initiatives to advance that objective. Nonetheless, immense challenges to the ambitious goal of eliminating disparities remain (Keppel, Pearcy, \& Wagener, 2002). Perhaps less recognized is the requisite need for a new paradigm given the immense challenges posed by the ambitious goal to eliminate disparities (Link, 1996) and the need to go beyond health care delivery (Levine at al., 2001).

Addressing the problem of population disparities has been limited by an insufficient definition of community. Indeed, epidemiology has been critiqued both for its inattention to important public health issues and the inadequacy of its consideration of community (Schwartz, Susser, \& Susser, 1999; Syme, 2004). Similar to community, the concepts of race/ethnicity have been given inadequate treatment. A dominant theme in social science is that race is not biological but is a social or cultural phenomenon (Freeman, 1998; King, 1997; King, Polednak, Bendel, Milsaint, \& Nahata, 2004). However, little explanation beyond "social" or "cultural" is provided. This paucity of explanation provides support for the notion that race is not a viable scientific construct and should be removed from social science analysis (Duster, 2001). Attempts to reconstruct it, such as replacing race/ethnicity with "racially classified social group" (King et al., 2004), is positive but insufficient. Alternatively, when race is supported, two critical errors are often committed: (a) race is viewed as an individual characteristic and (b) the analytic methods used in its assessment fail to consider the contextual nature of race, which in turn undermines the ability to discern the root causes of racial disparities (Jones, 2000).

A potential solution to these challenges has been described as a Community Development Model for Public Health Applications (Headen \& Robinson, 2001; Robinson, 2000). The Model uses a comprehensive construct for both community and race/ethnicity as the framework for defining disparities and developing initiatives to address those disparities effectively. For purposes of this Model, the definition of community encompasses the broad complexity and heterogeneity that characterize populations existing within national, 
regional, and local boundaries (Green \& Kreuter, 1999; Headen \& Robinson, 2001; Patrick \& Wickizer, 1995). History, culture, context, and geography provide the core basis for understanding the consciousness, experience, and social boundaries of communities. The following is a description of community that fits well into this Model:

It is the feeling of bonding, shared territory, or belonging that creates a community. Although physical and social boundaries appear static, they are always dynamically changing. Thus, the designations of neighborhood, block, division, housing unit, apartment building, and census tract become designations of communities within communities, similar to the concept of subcultures, or cultures within larger cultures. (Richards et al., 2002, p. 411)

Critical is the notion of shared consciousness. Labonte (1997) notes that communities are organized systems of people in relation to one another:

There is no "poor community" outside of poor persons coming together to share their experience and act upon transforming it. There is no "women's community" outside of two or more women sharing their reality, empowering themselves to act more effectively upon it. ... The essence of being a community is that there is something that is "shared." We cannot really say that a community exists until a group with a shared identity exists. (p. 90)

It has been noted that "every population has its own history, culture, organization, and economic and social divisions, which influence how and why people are exposed to particular factors" (Pearce, 1996, p. 682). Thus, the same determinants that form the basis for understanding community also mirror the consciousness, collective experience, and social boundaries of race/ethnic populations. Furthermore, race/ethnic communities are not homogeneous but are often composed of multiple populations each with particular histories, cultures, contexts, and geographies. The practitioner can choose to view a national population of any race/ ethnicity as reasonably homogeneous using the core determinants of history, culture context, or geography, or if necessary, can assess specific ethnic communities (i.e., Vietnamese, Cherokee, Puerto Rican, Jamaican, Italian) according to these same determinants. A core

\section{The Author}

Robert G. Robinson, Dr. PH, is the associate director for health equality at the Office on Smoking and Health, National Center for Chronic Disease Prevention and Health Promotion, Centers for Disease Control and Prevention (CDC) in Atlanta, Georgia. assumption within the Model is the influence of these core determinants on both community and race/ ethnicity. The assessment of these influences is indispensable to the successful application of the Model.

The Model adheres to certain principles and guided activities that are related to surveillance, research, planning, program and policy development, and evaluation. These principles and activities are (a) heterogeneity, diversity, and inclusivity; (b) a participatory approach; (c) development of trust; (d) community, race, and ethnicity; (e) services and programs; (f) policy and law; (g) community competence; (h) community development; (i) community prevention; and (j) comprehensiveness. These principles and activities encompass both process steps and related outcomes necessary for eliminating population disparities. Process steps include assessment of population groups, surveillance and evaluation, application of analytic rigor, representation, and fostering trust. Outcomes include services and programs, materials, policy and law, community competence, community development, and community prevention. The latter three also describe the three primary axes of the Model. The importance of comprehensiveness cannot be overstated because without a full range of applications, progress toward disparity elimination will be limited.

\section{AXIS I: COMMUNITY COMPETENCE}

Community competence evolves from perspectives related to cultural competence. Cultural competence has been variously described. It is defined as "ensuring that all health care providers can function effectively in a culturally diverse setting; it involves understanding and respecting cultural differences" (Randall, 2002, p. 59). It is offered as an assurance of diverse representation and accountability to race/ethnic communities (Richards et al., 2002). Appropriateness and sensitivity are substituted for competency. A review of cultural competence concluded that "no one has yet reviewed the literature and developed a core comprehensive approach to thinking about and implementing cultural competence in health care at multiple levels and from multiple perspectives" (Betancourt, Green, Carrillo, \& Ananeh-Firempong, 2003, p. 294).

Community competence, however, is a more focused approach that ideally reaches deeper into the population at risk with a core focus on applications. It avoids the ambiguity of being both a management tool for diversity and a guideline for program development. It avoids reductionism by making explicit that populations can be heterogeneous and are composed of more than the sum of their culture. It is compatible with a capacity-building construct because it is a problemsolving tool that is organically intertwined with local culture and values. (Hawe \& Shiell, 2000). It is an assessment that is grounded in primary and secondary constructs. The primary constructs are history, culture, context, and geography, and the secondary constructs 
are language, literacy, positive and salient imagery, multigenerational appeal, and diversity. The critical purpose is the development of research and program applications for a broad array of populations that are categorized as communities, groups, social strata, and individuals. Cultural competence is a construct that is best utilized when the critical focus is the individual. Alternatively, community competence improves in saliency the higher the level of aggregation in the population being addressed. The Model assumes that the higher the level of organization (community in contrast to individuals), the more inputs from the Community Competent Axis will be required. However, the Model is not zero-sum. No population loses; rather, it is a matter of degree and intensity resulting in the most effective outcome for the community, or group, or strata, or indi$\operatorname{vidual}(\mathrm{s})$.

\section{Community Competence: Primary and Secondary Constructs}

The primary constructs provide the foundation of community competence because it is through these mechanisms that the collective consciousness, experience, and social boundaries of populations evolve. In addition, historical, cultural, contextual, and geographical experiences "remain exceedingly powerful predictors of the health status of human populations" (Loomis \& Wing, 1990, p. 2). History captures the important phenomena that have shaped the collective consciousness of a people, that is, slavery for African Americans, the Holocaust for Jews, and near-genocide for Native Americans. Culture includes the values, norms, faiths, and beliefs that inform the behaviors and collective lifestyles of a people. Context is the current reality that confronts a people, that is, adequacy of health care, stresses resulting from racism or sexism or homophobia, and conditions of employment or housing or education. Geography provides an ecologic mechanism for distinguishing within and across populations. For example, it helps to distinguish the poor White communities of Appalachia from those of the Midwest or Black people living in New York City from those residing in Atlanta. Although race/ethnic populations share commonalities within a national community, geographic parameters help frame and may even determine unique differences that can much more effectively be addressed by the Model that focuses on community competence and explicitly addresses matters of diversity.

The secondary constructs of community competencelanguage, literacy, positive and salient imagery, multigenerational appeal, and diversity-also are indispensable to effective interventions for disparities. Provision of language or interpreter services is a key aspect of assuring competent services (Chang \& Fortier, 1998) and is not a trivial issue given that more than one in five patients from communities of color have language difficulties when receiving care (Brach \& Fraser, 2000).
Developing low literacy materials also has proven to be effective (Jacobson et al., 1999). Positive imagery reflects the strengths and assets of a population and salient imagery focuses on those images that are particularly effective with a population. A multigenerational approach ensures that interventions will not overlook the potential importance of targeting more than one generation or stressing the importance of one generation (i.e., youth) as an important reason for behavior change among adults. Diversity encompasses a range of race/ ethnicity and other sociodemographic (i.e., rural/urban, poverty) variability contained within a population, including gender orientation.

The protocol of community competence is achieved by assessing each population and considering each intervention in relation to the primary and secondary constructs (see Figure 1). Consideration will encompass developing a new intervention, tailoring an existing intervention, or assuring whether an existing intervention is appropriate for a specific population. The protocol is dynamic and relative because the determinants of history, culture, context, and geography are neither static nor of the same relevance to different populations. For example, geography and culture by necessity influence tobacco control initiatives for some Native American tribes in the United States because tobacco has traditional and spiritual importance in ceremony and ritual. Interventions designed for these tribes must be sensitive to their traditions. However, for Alaska natives, which comprise a portion of the more than 500 tribes among Native Americans, the tobacco leaf has no comparable importance.

\section{AXIS 2: COMMUNITY DEVELOPMENT}

Strategic planning efforts to eliminate population disparities that include an emphasis on community development (i.e., capacity and infrastructure + social capital) rely on an understanding of community. This is distinct from planning and problem solving that relies on epidemiologic and statistical methods that disaggregate because their purpose is to determine risk factors related to populations and/or individuals. Indeed, it is insufficient for the core client of public health to be the individual, even if they are massed into numbers justifying a designation of population-based science and practice (Benatar, 2003; Robinson, 2004).

Traditional epidemiologic methods fall short because they primarily measure certain group characteristics and fail to identify other relevant characteristics that may exist within a specific community. The challenge for public health is to have available the appropriate quantitative and qualitative models and methods that allow for deeper rather than broader assessment and analysis within at-risk populations (Michael, 1999). Deeper assessments are inclusive of complexity and equate with systems-based models that reflect the observable world and not statistical estimates 
of risks of the unobservable (Michael, 1999). In essence, they are models that encompass whole populations and not disaggregated segments that are prioritized based on risk assessments.

Community development comprises two components: (a) capacity and infrastructure and (b) social capital. The constructs for these components were distilled from an extensive review of the literature whose aim was to identify and define the dimensions of community capacity (Goodman et al., 1998). In addition, it addresses a weakness in the social capital literature as reflected in public health to emphasize relational or process indicators and ignore the impact of material or infrastructure on health outcomes (Hawe \& Shiell, 2000). Of interest, even though social capital was defined in terms of process, community residents specified the importance of infrastructure when being interviewed (Ziersch, Baum, MacDougall, \& Putland, in press). A

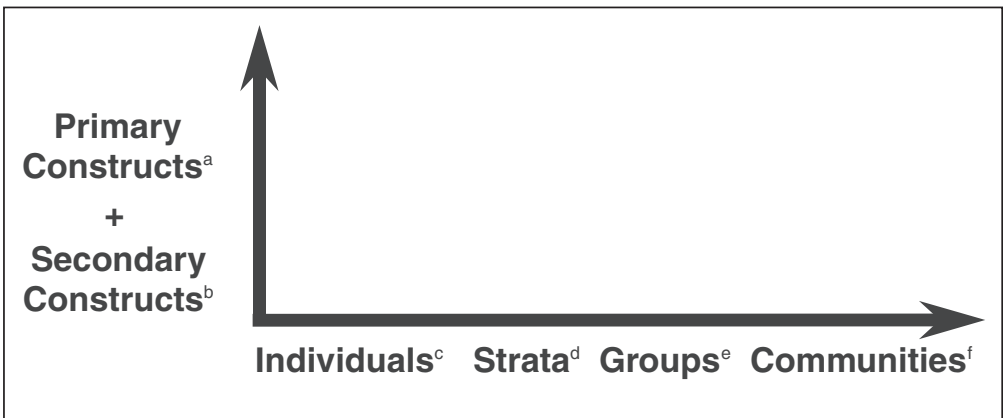

FIGURE 1 Community Competence

a. History, culture, context, geography.

b. Language, literacy, positive imagery, salient imagery, multigenerational, diversity.

c. Person, primary family.

d. Socioeconomic status, gender, literacy.

e. Drug treatment centers, physically challenged, prisons, cancer patients/ survivors.

f. American Indian/Alaska Native (AI/AN), Asian American/Pacific Islander (AA/PI), Black, Hispanic/Latino (H/L), White, Gay/Lesbian/Bisexual/ Transgender (GLBT), religion.

consistent weakness of social capital reflected in an extensive review (Hawe \& Shiell, 2000) is the tendency to equate it with material capacity, resulting in conceptual weaknesses. Indeed, "supportive relational ties are not a sufficient antidote to material deprivation and learned helplessness" (Hawe \& Shiell, 2000, p. 879). This Model offers that capacity and infrastructure and social capital are distinct and complementary and are best subsumed within the construct of community development. Similar to community competence, the degree to which community development inputs are needed is related to whether a population is organized as a community, group, strata, or individual(s) (see Figure 2).

\section{Community Development: Constructs}

The constructs of capacity and infrastructure include research and researchers, programs and services, leaders, organizations, and networks. A community will possess capacity and infrastructure to the degree there is, first, research that addresses that community's specific needs or produces specific information and, second, researchers who are representative of the community; programs and services that are community competent and enable delivery of services to address needs; leaders who are knowledgeable and serve as advocates as well as decision makers; organizations that represent the population's interests and provide relevant resources and services; and networks that provide logistical support for strategic planning, information sharing, priority setting, and policy development. Capacity and infrastructure are enhanced when other tangible resources, such as consultants, technical assistance, and training programs, are available.

The ability of a community to access its resources and engage in interactive processes is directly depend- ent on the level of cooperation, collaboration, reciprocity, trust, and social cohesion that should result for the optimal development and implementation of programs and related strategies (Goodman et al., 1998; Hawe \& Shiell, 2000; Kennedy, Kawachi, Prothrow-Stith, Lochner, \& Gupta, 1998; Oetting et al., 1995). This is, in essence, a community's social capital. Social capital is distinguished from capacity and infrastructure in that it addresses the processes by which respective agents interact with and engage one another rather than the material condition of a community. It is explicitly focused on a community's assets rather than deficits (Yen \& Syme, 1999). It also provides a framework for understanding a community's stage of readiness to address public health problems. In general, technology and availability of funding, staffing, and responsive political institutions can enhance the social capital of a community (Goodman et al., 1998). The interrelation of social capital with capacity and infrastructure (i.e., leaders, networks) is evident in the fact that the degree to which power and influence is present may reflect the level of existing social capital (Goodman et al., 1998). The Model prioritizes capacity and infrastructure relative to social capital because without the former a population will have to rely on processes to recruit and secure aid from the external environment.

\section{AXIS 3: COMMUNITY PREVENTION, PREVENTION, AND CONTROL APPLICATIONS}

Community prevention, prevention, and control provide a matrix along which all "categorical" populations can be placed. Community prevention broadens traditional public health approaches of prevention and control to include an explicit focus on community, reflecting the underpinnings of history, culture context, and 
geography, in contrast to populations defined either as groups or strata or individuals. Prevention includes primary, secondary, and tertiary approaches (Levine et al., 2001), including environmental efforts that are intended for all citizens. In tobacco control, for example, prevention would include comprehensive countermarketing campaigns, excise taxes, and regulatory efforts related to advertising and clean indoor air laws. Control includes interventions focused on cure, care, and quality of life. Prevention and control also are determined by time. If the intervention is early and successful, then some event has been prevented. Control strategies occur downstream or later in time and are intended to lessen the consequences of an event or to provide a cure. In essence, community prevention is additive to prevention and control and assures an explicit focus on community and rigorous attention to community competence and community development. If viewed according to a criteria of comprehensive, community prevention, prevention and control comprise interrelated intervention components reflecting a systemic approach to populations and the goal of defining and eliminating disparities.

Community prevention is specific to populations organized as communities and explicitly factors in their determinants that shape their collective consciousness and experience. Communities will have a level of systemic organization that is less evident in groups, strata, or individual(s). Collectively, the three modes of applications combine and achieve a comprehensive public health effort to define and eliminate population disparities. Comprehensiveness also provides a marker for distinguishing between approaches focused on a medical or health care delivery model in contrast to a public health paradigm (Greenspan, 2001). Ideally, the most effective efforts related to elimination of disparities will occur within the intersection of the respective intervention modalities with focused applications that target all respective populations (i.e., individuals, strata, group, community) (see Figure 3).

\section{SUMMARY OF MODEL}

The community model to eliminate population disparities combines three primary axes: community competence, community development, and community prevention. These axes and their relationship to respective communities, groups, strata, and individuals are represented in Figure 4.

Populations as represented by individuals, strata, groups, and community are shown in relationship to community competence and community development within the respective modalities of control, prevention, and community prevention. The figure reflects the level of intensity, influenced by the degree of complexity of the respective populations associated with their organizational characteristics. The guiding principal is that communities are more complex than groups, strata, and individuals. Complexity is not a measure of diversity. For example, strata are more likely to reflect a broad range of diversity when compared to groups. Rather, complexity reflects the extent to which a population's consciousness, collective experience, and social boundaries are shaped by history, culture, context, and geography. Individuals are dispersed and can be members of several communities. Strata are typically characterized by singularly important sociodemographic characteristics such as income or education. Strata will reflect the diversity of the society in which they evolve. Groups are more complex because they are more accessible to the determinants of history, culture, context, and geography that result in a greater degree of homogeneity. It is a homogeneity with the potential of counteracting race/ ethnic diversity. For example, cancer patients or survivors or veterans of a particular war or persons in drug rehabilitation can be reached as a group and because of the power of their experience may achieve a high quality of homogeneity. This is less possible for strata who may achieve homogeneity only in the most extraordinary of circumstances. For example, a nation will be unified when facing a recognized external threat. Strata will respond similarly given an analogous situation.

The figure illustrates that the intensity or allocation of specific interventions will increase according to the complexity of the population. In addition, control and prevention interventions are cumulative, with the most comprehensive effort occurring within the construct of community prevention. Community competence, community development, and community prevention will be greater for communities, followed by groups, strata, and individuals. Individuals, strata, and groups will necessarily be reached with specific interventions if warranted, but they also will be reached as in their community. It is possible that an individual may not iden- 
tify with a specific community, have a lifestyle that is nonreliant on a community identity, and may have multiple community identities and related influences. This is not a critical factor because the problem being addressed by the Model is not individual well-being but eliminating population disparities. If disparity elimination is to occur then the critical focus of the interventions must be the community, with sufficient attention provided to groups, strata, and individuals. In essence, the error associated with missing an individual is of lesser significance than if it was a community that was missed. Of critical importance is the assumption that the Model makes explicit the importance of multiple approaches and populations. The Model acquires its effectiveness from the explicit attention to comprehensive approaches to reach communities, with priority attention to their diversity and substantive attention to the groups, strata, and individuals that comprise the total society.

The Model's applicability is not lessened by a mix of personal identities or associations. For example, if an initiative is designed to address the Gay/Lesbian/Bisexual/ Transgender (GLBT) community, it will also be possible to reach GLBT individuals of different races and ethnicity within their respective communities. The practitioner will choose whether to reach a "national community" of GLBT, or to factor in race/ethnicity and reach them separately, or to combine the respective approaches. Indeed, the Model encourages an approach that explicitly targets multiple populations and/or individuals in multiple settings. However, the final decision regarding a strategic plan and implementation will reflect availability of resources, the problem being addressed, degree of complexity, and the existing state of the art regarding best and/or promising practices as they relate to community, group, strata, or individual(s).

Women, who are diverse racially and sociodemographically, also may be targeted for initiatives through community, groups, and strata. The Model allows for flexibility because the underlying determinants of community and race/ethnicity are relative and dynamic, changing in accordance with the particular population, how it is organized, and the problem being addressed. In essence, utilizing history, culture, context, and geography to understand the target population requires full appreciation of the axiom that "one shoe does not fit all."

Strategic plans to reach groups, social strata, and individuals are equally served by the Model. A group may be a prison population or residential programs for substance abuse. Contextual and geographic issues may emerge as the critical focus when evaluating the basis for competent program development, along with the array of secondary constructs. Community development may be less needed. However, development of leaders and organizations to serve will likely still be important. Similarly, strata such as the poor or bluecollar workers will represent a compelling picture of diversity. The practitioner can devise strategic interventions that target specific strata (i.e., health insurance coverage or excise taxes) or reach individuals (i.e., clinical and behavior change protocols) within their specific race/ethnic communities. Again, final decisions will be influenced by resources, problem specificity, complexity, and the state-of-the-art of interventions. The Model makes explicit that interventions are available to reach an individual but the underlying assumption is that successful efforts to eliminate disparities will occur only in the presence of comprehensive efforts reaching all populations, but most important, respective communities.

\section{CASE EXAMPLE}

An effective example of the Model in action is Pathways to Freedom (Robinson, Sutton, James, \& Orleans, 2003), a tobacco cessation guide produced for the African American community in 1992 and recently updated in 2003. The Pathways to Freedom guide was designed as a community-competent guide to address cessation needs from both an individual and community perspective. It addresses first the unique historical relationship that African Americans have had with tobacco since the time of slavery in the nation when it was a key 


\begin{tabular}{|c|c|c|c|c|c|c|}
\hline & \multicolumn{2}{|c|}{ CONTROL $^{a}$} & \multicolumn{2}{|c|}{ PREVENTION ${ }^{b}$} & \multicolumn{2}{|c|}{$\begin{array}{l}\text { COMMUNITY } \\
\text { PREVENTION }^{\circ}\end{array}$} \\
\hline & $\begin{array}{l}\text { COMMUNITY } \\
\text { COMPETENCE }\end{array}$ & $\begin{array}{c}\text { COMMUNITY } \\
\text { DEVELOPMENT }\end{array}$ & $\begin{array}{c}\text { COMMUNITY } \\
\text { COMPETENCE }\end{array}$ & $\begin{array}{c}\text { COMMUNITY } \\
\text { DEVELOPMENT }\end{array}$ & $\begin{array}{l}\text { COMMUNITY } \\
\text { COMPETENCE }\end{array}$ & $\begin{array}{c}\text { COMMUNITY } \\
\text { DEVELOPMENT }\end{array}$ \\
\hline INDIVIDUAL $^{d}$ & $x$ & $x$ & $x$ & X & $X X$ & $X X$ \\
\hline STRATA $^{e}$ & $X X$ & $X X$ & $X X$ & $X X$ & $X X X X$ & $X X X X$ \\
\hline GROUP $^{f}$ & $X X X$ & $X X X$ & $X X X$ & $X X X$ & $\begin{array}{l}X X X \\
X X X\end{array}$ & $\begin{array}{l}X X X \\
X X X\end{array}$ \\
\hline COMMUNITY $^{g}$ & $X X X X$ & $X X X X$ & $X X X X$ & $X X X X$ & $\begin{array}{l}X X X X \\
X X X X\end{array}$ & $\begin{array}{l}X X X X \\
X X X X\end{array}$ \\
\hline
\end{tabular}

FIGURE 4 Model to Eliminate Population Model Disparities

a. Treatment, pharmaceutical, counseling.

b. Policy, education, counter marketing, immunization.

c. Control + prevention.

d. Person, primary family.

e. Socioeconomic status, gender, literacy.

f. Drug treatment centers, physically challenged, prisons, cancer patients/survivors.

g. American Indian/Alaska Native (AI/AN), Asian American/Pacific Islander (AA/PI), Black, Hispanic/Latino (H/L), White, Gay/Lesbian/ Bisexual/Transgender (GLBT), religion.

agricultural cash crop. It acknowledges that African Americans are especially targeted by the tobacco industry bent on exploiting the yearnings and frustrations of this community. Careful effort was given to ensure that the guide was written with appropriately positive and salient images and a targeted literacy level no higher than 7 th grade.

Most important, Pathways to Freedom facilitated community development by involving multiple facets of the African American leadership and organizations in addressing tobacco use. For example, the guide was distributed through churches as well as through physicians' offices and other health care facilities. It helped facilitate the emergence of leaders that diversified the tobacco control movement. Pathways to Freedom is equally useful for initiatives targeted toward a local urban neighborhood, a regional African American sorority or fraternity, and a national civil rights organization (Headen \& Robinson, 2001). Finally, Pathways to Freedom is considered to be part of a comprehensive array of interventions and initiatives such as community development, advocacy, and excise taxes that assisted in the elimination of tobacco-use disparity between Blacks and Whites (Carmona et al., 2004; Woollery, Trosclair, Husten, Caraballo, \& Kahende, 2003). This conclusion supports the finding that con- text as measured by social cohesion affects smoking prevalence and that tobacco use is more than the sum of individual behavior (Patterson, Eberly, Ding, \& Hargreaves, 2004).

\section{CHALLENGES TO THE MODEL}

There are several barriers to adopting the communityfocused Model (Headen \& Robinson, 2001). First is that epidemiologic methods of defining and measuring variables such as race/ethnicity and socioeconomic status must be overlaid with more focused assessment and analysis of relevant characteristics that may exist within at-risk populations. This increasingly detailed analysis, needing both quantitative and qualitative methods, requires additional resources both in funding and in personnel. A combination of quantitative and qualitative methods is most appropriate when problems evolve from social context and require broad system change (Ratcliffe, 1978). Commitment of needed resources is especially difficult in the current budget environment confronting most federal and state public health programs. Application of the Model requires evaluation of opportunity costs, or the potential downside of spending on one venture only to facilitate 
another negative outcome elsewhere (Oliver, Healey, \& Le Grand, 2002).

Another barrier to the community-focused Model is the complex debate regarding race as a social or biological construct. Population disparities are primarily determined structurally, not biologically. Disparities are interrelated and result largely from multiple factors such as health, education, housing, employment, and justice. Yet, agencies are rarely equipped to approach solutions in a manner that is multisectorial. Such a cross-cutting approach is essential to effective application of the Model. The importance of accessing communities, often shaped by race/ethnicity, only adds to the challenge.

Analytic methods also are limited in their approach to the assessment of race/ethnicity. Typically, race/ ethnicity will lose importance when multivariate analysis results in nonsignificance, usually concluding that the important predictor is income or education. However, this is inherently reductionist and negates the importance of race/ethnicity when developing or tailoring interventions. Also, race/ethnicity is best viewed as a contextual variable and is not a characteristic of the person (Jones, 2000). This is further compounded by racism, which contributes to social structure inequities that give rise to population disparities (Cohen \& Northridge, 2000). Consistent wih the assumptions of the model, analytic rigor requires two levels of analysis. The first level assesses race/ethnicity as a risk factor and ascertains its etiologic role. The second level assesses race/ethnicity in regard to relationship to evolving interventions or implementation of existing interventions. Race/ethnicity may not prove to be a significant predictor of a health-related outcome, particularly given the descriptive nature of the variable, but it will remain salient at the level of community when social context is explicit and strategic efforts are engaged to eliminate a disparity.

A final challenge will be failure to utilize a breadth of indicators to determine disparities. In tobacco control, this is best illustrated by the need to rely on more than prevalence. The definition, formulated at the National Conference on Tobacco and Health Disparities (NCTHD) held in December 2002, provides a useful example:

... differences in the patterns, prevention, and treat-
ment of tobacco use; the risk, incidence, morbidity,
mortality, and burden of tobacco-related illness that
exist among specific population groups in the United
States; and related differences in capacity and infra-
structure, access to resources, and environmental
tobacco smoke exposure. (Fagan et al., 2004, p. 211)

Using only one indicator will limit comprehensive planning efforts either because of the absence of quantitative data for certain populations or the indicator is an insufficient marker to represent the total condition of disparity experienced by a population. For example, a community may experience historical high levels of tar- geting by the tobacco industry and exclusion from the tobacco control movement with the result that there are limited resources available to that community with which to address the problem. Breadth of indicators allows for the inclusion of a population and implicitly creates a place at the table for them to ascertain and impact their interests.

\section{SUMMARY}

There is considerable consensus that disparities are a social ill that are destructive of life and welfare and ultimately of the social contract that binds the nation together as a collective. The public health community has rightly committed to eliminating disparities and has made some progress in advancing this goal. Resources, strategy, and cooperation have been characterized as keys in health promotion initiatives in diverse and lowincome populations (Kerner, Dusenbury, \& Mandelblatt, 1993). Yet, resources, more than theory and methodology, may be the ultimate challenge and the most resistant barrier to a comprehensive effort to eliminate population disparities. Clearly, however, a framework that enables a more focused and thus more intense application of resources within at-risk populations is needed if additional gains in eliminating healthrelated disparities are to be realized. A Model based on community rather than traditional reductionist approaches may begin to affect those more intractable disparities that remain, particularly those heavily influenced by poverty and racism. Interventions that reach deeper into at-risk populations and use a comprehensive strategic plan to focus scarce resources where they can do the most good will facilitate empowerment and intersectoral planning to address problems that are embedded in social structure and the legacy of social injustice.

\section{REFERENCES}

Benatar, S. R. (2003). Bioethics: Power and injustice. IAB presidential address. Bioethics, 17(5-6), 387-398.

Betancourt, J. R., Green, A. R., Carrillo, J. E., \& Ananeh-Firempong, O, II. (2003, July-August). Defining cultural competence: A practical framework for addressing racial/ethnic disparities in health and health care. Public Health Reports, 118, 293-302.

Brach, C., \& Fraser, I. (2000, November). Can cultural competency reduce racial and ethnic health disparities? A review and conceptual model. Medical Care Research and Review, 57(Suppl. 1), 181221.

Carmona, R., Gfroerer, J., Caraballo, R., Yee, S. L., Husten, C., Pechacek, T., et al. (2004, January 30). Prevalence of cigarette use among $14 \mathrm{racial} / \mathrm{ethnic}$ populations-United States, 1999-2001. Morbidity and Mortality Weekly Report, 53(3), 49-52.

Chang, P. H., \& Fortier, J. P. (1998). Language barriers to health care: An overview. Journal of Health Care for the Poor and Under Served, 9(Suppl.), S5-S20.

Cohen, H. W., \& Northridge, M. E. (2000). Getting political: Racism and urban health. American Journal of Public Health, 90, 841-842.

Duster, T. (2001, September 14). Buried alive: The concept of race in science. Chronicle of Higher Education. 
Fagan, P., King, G., Lawrence, D., Petucci, S. A., Robinson, R. G., Banks, D., et al. (2004, February). Eliminating tobacco-related health disparities: Directors for future research. American Journal of Public Health, 92(2), 211-217.

Freeman, H. P. (1998). The meaning of race in science-considerations for cancer research: Concerns of special populations in the national cancer program. Cancer, 82(1), 219-225.

Goodman, R. M., Speers, M. A., McLeroy, K., Fawcett, S., Kegler, M., Parker, E., et al. (1998, June). Identifying and defining the dimensions of community capacity to provide a basis for measurement. Health Education \& Behavior, 2(33), 258-278.

Green, L. W., \& Kreuter, M. W. (1999). Health promotion planning: An educational and ecological approach (3rd ed.). Mountain View, CA: Mayfield.

Greenspan, B. (2001, September-October). Health disparities and the U.S. health care system. Public Health Reports, 116, 417-418.

Hawe, P., \& Shiell, A. (2000). Social capital and health promotion: A review. Social Science \& Medicine, 51, 871-885.

Headen, S. W., \& Robinson, R. G. (2001). From slavery to addiction: African Americans and tobacco. In R. L. Braithwaite \& S. E. Taylor (Eds.), Health issues in the Black community (2nd ed., pp. 347383). San Francisco: Jossey-Bass.

Jacobson, T. A., Thomas, D. M., Morton, F. J., Offutt, G., Shevlin, J., \& Ray, S. (1999, August 19). Use of a low-literacy patient education tool to enhance pneumococcal vaccination rates: A randomized control trial. Journal of the American Medical Association, 282(7), 646-650.

Jones, C. P. (2000). Invited commentary: "Race," racism, and the practice of epidemiology. American Journal of Epidemiology, 154(4), 299-304.

Kennedy, B. P., Kawachi, I., Prothrow-Stith, D., Lochner, K., \& Gupta, V. (1998). Social capital, income inequality, and firearm violent crime. Social Science \& Medicine, 47, 7-17.

Keppel, K. G., Pearcy, J. N., \& Wagener, D. K. (2002, January). Trends in racial and ethnic-specific rates for the health status indicators: United States, 1990-1998. Statistical News, 23, 1-16.

Kerner, J. F., Dusenbury, L., \& Mandelblatt, J. S. (1993). Poverty and cultural diversity: Challenges for health promotion among the medically underserved. Annual Review of Public Health, 14, 35577.

King, G. (1997). The "race" concept in smoking: A review of the research on African Americans. Social Science Medicine, 45(7), 1075-87.

King, G., Polednak, A., Bendel, R. B., Milsaint, M. C., \& Nahata, S. B. (2004). Disparities in smoking cessation between African Americans and Whites: 1990-2000. American Journal of Public Health, 94(11), 1965-1971.

Labonte, R. (1997). Community, community development, and the forming of authentic partnerships: Some critical reflections. In M. Minkler (Ed.), Community organizing and community building for health (pp. 88-101). New Brunswick, NJ: Rutgers University Press.

Levine, R. S., Foster, J. E., Fullilove, R. R., Fullilove, M. T., Briggs, N. C., Hull, P. C., et al. (2001, September-October). Black-White inequalities in mortality and life expectancy, 1933-1999: Implications for healthy people 2010. Public Health Reports, 116, 473-483.

Link, B. G. (1996, April). Editorial: Understanding sociodemographic differences in health-The role of fundamental social cause. American Journal of Public Health, 86(4) 471-472.

Loomis, D., \& Wing, S. (1990). Is molecular epidemiology a germ theory for the end of the Twentieth Century? International Journal of Epidemiology, 19(1), 1-3.
Michael, A. J. (1999, May 15). Prisoners of the proximate: Loosening the constraints on epidemiology in an age of change. American Journal of Epidemiology, 149(10), 887-897.

Oetting, E. R., Donnemeyer, J. F., Plested, B. A., Edwards, R. W., Kelly, K., \& Beauvals, F. (1995). Assessing community readiness for prevention. International Journal on the Addictions, 30(6), 659-683.

Oliver, A., Healey, A., \& Le Grand, J. (2002, August 17). Addressing health inequalities. Lancet, 360(9332), 564-567.

Patrick, D. L., \& Wickizer, T. M. (1995). Community and health. In B. C. Amick, S. Levine, A. R. Tarlov, \& D. C. Walsh (Eds.), Society and health. New York: Oxford University Press.

Patterson, J. M., Eberly, L. E., Ding, Y., \& Hargreaves, M. (2004). Associations of smoking prevalence with individual and area level social cohesion. Journal of Epidemiology Community Health, 58, 692-697.

Pearce, N. (1996, May). Traditional epidemiology, modern epidemiology, and public health. American Journal of Public Health, 86(5), 678-683.

Randall, V. R. (2002, Fall). Racial discrimination in health care in the United States as a violation of the international convention on the elimination of all forms of racial discrimination. Journal of Law and Public Policy, 14(1), 46-91.

Ratcliffe, J. A. (1978). Population control versus social reorganization: The emergent paradigm. International Journal of Health Services, 8(3), 559-568.

Richards, L., Kennedy, P. H., Krulewitch, C. J., Wingrove, B., Katz, K., Wesley, B., et al. (2002, July). Achieving success in poor urban minority community-based research: Strategies for implementing community-based research within an urban minority population. Health Promotion Practice, 3(3), 410-420.

Robinson, R. G. (2000). Eliminating population disparities in tobacco control: SmokeLess States Tobacco News. American Medical Association, 6(1), 5.

Robinson, R. G. (2004, April). Response to S. Leonard Syme's essay [Letter to the editor]. Preventing Chronic Disease. Retrieved from http://www.cdc.gov/pcd/issues/2004/apr/04_0005.htm.

Robinson, R. G., Sutton, C., James, D., \& Orleans, T. C. (2003). Pathways to freedom: Winning the fight against tobacco (2nd ed.). Atlanta, GA: Centers for Disease Control and Prevention.

Schwartz, S., Susser, E., \& Susser, M. A. (1999). A future for epidemiology? Annual Review of Public Health, 20, 15-33.

Syme, S. L. (2004, January). Social determinants of health: The community as an empowered partner. Preventing Chronic Disease. Retrieved from http:// www.cdc.gov/pcd/issues /2004/jan/ syme.htm

U.S. Department of Health and Human Services. (2000). Tracking healthy people 2010. Washington, DC: Government Printing Office.

Woollery, T., Trosclair, A., Husten, C., Caraballo, R., \& Kahende, J. (2003, October 10). Cigarette smoking among adults-United States, 2001. Morbidity and Mortality Weekly Report, 52(40), 943956.

Yen, I. H., \& Syme, S. L. (1999). The social environment and health: A discussion of the epidemiologic literature. Annual Review of Public Health, 20, 287-308.

Ziersch, A. M., Baum, F. E., MacDougall, C., \& Putland, C. (in press). Neighborhood life and social capital: The implications for health. Social Science \& Medicine. 